D.O.I. $10.3895 / \mathrm{S} 1808-04482006000400009$

\title{
AS ABORDAGENS DE CLUSTERS E DE SISTEMAS DE INOVAÇÃO: MODELO HIIBRIDO DE ANÁLISE DE AGLOMERAÇÕES INDUSTRIAIS TECNOLOGICAMENTE DINÂMICAS
}

\section{THE CLUSTERS AND INNOVATION SYSTEMS APPROACHES: AN HYBRID MODEL TO ANALYZE TECHNOLOGICALLY DYNAMICS INDUSTRIAL AGGLOMERATIONS}

\author{
Bruno dos Santos Silvestre ${ }^{1}$; Paulo Roberto Tavares Dalcol ${ }^{2}$ \\ ${ }^{1}$ Centrais Elétricas Brasileira S.A. - Eletrobrás - Rio de Janeiro - Brasil bruno.silvestre@eletrobras.com \\ ${ }^{2}$ Pontifícia Universidade Católica do Rio de Janeiro - PUC-Rio - Rio de Janeiro - Brasil \\ prtd@,ind.puc-rio.br
}

\begin{abstract}
Resumo
Este trabalho visa apresentar um referencial teórico inovador, elaborado para a análise de aglomerações industriais que apresentem um conteúdo tecnológico significativo. Uma revisão bibliográfica das abordagens de clusters e sistemas de inovação é realizada, onde se identificam os principais problemas de cada uma das abordagens e as contribuições para este campo de estudo tão dinâmico e promissor. Como principal resultado deste trabalho, elaborou-se um novo modelo teórico, um modelo híbrido, para análise de aglomerações, que utiliza elementos das abordagens de clusters e de sistemas de inovação, consistindo em uma interessante ferramenta de análise. Da abordagem de clusters, o relacionamento entre os atores são destacados por meio de suas conexões de conhecimento, que no seu conjunto formam o sistema de conhecimento. Da abordagem de sistemas de inovação utiliza-se as vertentes de sistemas setoriais e tecnológicos de inovação, buscando-se os aspectos relacionados ao desenvolvimento de capacitações tecnológicas, mudanças tecnológicas e inovação. O modelo desenvolvido neste trabalho foi aplicado na aglomeração industrial de petróleo e gás da região produtora da Bacia de Campos gerando resultados promissores.
\end{abstract}

Palavras-chave: aglomerações industriais; clusters; sistemas de inovação.

\section{Introdução}

O presente trabalho consiste em um estudo eminentemente teórico e está centrado nas abordagens de clusters e de sistemas de inovação (setoriais e tecnológicos). A escolha dessas abordagens se deve ao fato de possuírem uma maior correlação com a aglomeração industrial na qual foi aplicada esta metodologia e por apresentarem um retrospecto recente de dinamismo e consolidação. Além disso, este esforço parte no sentido de buscar uma metodologia de análise 
voltada para aglomerações industriais tecnologicamente dinâmicas e que ofereça alternativas para os estudantes e pesquisadores do tema.

Para isso, uma revisão bibliográfica é feita nas seções 2 e 3, para posteriormente se fazer a apresentação do Modelo Híbrido proposto. Cabe ressaltar que o referido modelo já foi testado na aglomeração industrial de petróleo e gás da região produtora da Bacia de Campos, mostrando resultados promissores (SILVESTRE, 2006).

\section{A Abordagem de Clusters}

Do trabalho precursor de Alfred Marshall (1920) até os dias de hoje, muitos estudos referentes às externalidades dos aglomerados têm sido produzidos no mundo todo. Pesquisadores e homens públicos têm classificado os aglomerados por diferentes nomenclaturas ao longo desse tempo, tais como, distritos industriais, milieus, clusters, redes, arranjos, sistemas e outras.

De acordo com a metáfora 'Marshalliana', freqüentemente utilizada por diversos estudiosos atuantes na área de aglomerados industriais, 'o conhecimento está no ar' e a absorção desse conhecimento pelas firmas agrupadas é um processo natural, não exigindo esforço explícito para sua absorção, que fica, portanto, livremente disponível como um bem público. Isso significa dizer que, para esses autores, nos aglomerados de firmas existe um tipo de conhecimento, independente de ser adquirido ou não, que está no ar, na atmosfera, portanto, disponível. (GIULIANI, 2004).

Marshall (1920: 271) define essa característica como uma 'atmosfera industrial', na qual todas as firmas aproveitam dessa vantagem, por estarem localizadas dentro de um aglomerado. Para ele, "os mistérios dos negócios tornaram-se não-mistérios, permanecendo no ar, e as crianças aprendem muitos deles inconscientemente".

Seguindo essa linha de raciocínio, Porter (1990), em seu famoso livro "A Vantagem Competitiva das Nações", popularizou o conceito de cluster industrial nos círculos industrial, acadêmico e político. Daí em diante, o conceito de cluster tornou-se 'palavra mágica' nesses círculos e um grande número de adeptos começou a trabalhar com o referido conceito. Nesse contexto, estudos teóricos e empíricos sob a abordagem de cluster industrial são fáceis de serem encontrados. No entanto, essa popularização produziu alguns problemas centrais em uma parte da literatura: a multiplicidade de nomenclaturas, expressões e tipologias de cluster, conforme se discute a seguir.

O conceito de cluster está relacionado a uma concentração geográfica de firmas em um ou mais setores correlatos ou indústrias complementares (não necessariamente na mesma indústria ou setor) e espacialmente concentradas. Pesquisadores, trabalhando em estudos relacionados a cluster, geralmente dão especial atenção à proximidade territorial, com a qual as firmas operam. Estes 
aglomerados situam-se no mesmo espaço geográfico, que pode estar delimitado por uma única cidade, uma região, um estado ou mesmo um país (ALBU, 1997 e BASANT, 2002).

As conceituações de clusters encontradas na literatura podem variar de forma bastante intensa. Malmberg (2003) concorda com este ponto de vista e argumenta que em virtude da má utilização do conceito de cluster e da falta de critérios no uso da palavra, a adoção do termo leva a alguns pré-requisitos, que muitas vezes, na prática, não são verdadeiros.

Para Albu (1997), os clusters podem ser conceituados, além do aspecto da aglomeração física, por sua especialização produtiva e pela existência de uma rede de relacionamentos entre firmas, que pode ser de natureza mais ou menos complexa e, conseqüentemente, mais ou menos dinâmica e geradora de vantagens competitivas para as firmas, a qual servirá de base para o escopo deste trabalho.

Outro fator problemático, além da questão da conceituação geral, consiste na multiplicidade de tipologias e classificações de clusters. A existência de diversos tipos de clusters e a verificação desse aspecto, na prática, pelos pesquisadores fez com que esse problema se agravasse.

Algumas das diferentes tipologias encontradas na revisão da literatura sobre clusters podem ser verificadas, em trabalhos como os de: Amin (1994), Humphrey (1995), Markusen (1996), Storper (1997), Cassiolato e Lastres (2001), entre outros.

Para Marceau (1994), os clusters podem apresentar algumas vantagens para as firmas agrupadas, tendo em vista que, por estarem interligadas, teoricamente encorajariam os fluxos de informação e a colaboração entre elas. Alguns estudos empíricos confirmam esta suposição, muito embora se trate de aglomerações específicas em países intensamente inovadores do Hemisfério Norte (Canadá e Alemanha), tais como Audretsch e Feldman (1996), Bender et. al., (2002) e Aharonson et. al. (2004). De qualquer forma estes três trabalhos também identificam algumas externalidades negativas que, em certos casos, podem ser desenvolvidas e até superar as externalidades positivas provenientes da aglomeração de firmas.

No entanto, outro grande bloco da literatura ressalta evidências empíricas que apontam que a atividade econômica agrupada geograficamente de um setor particular por si só não representa nenhuma vantagem para as firmas ali localizadas. Portanto, para eles, se a teoria se apresenta promissora, o mesmo não acontece nos estudos empíricos, que mostram resultados desapontadores. Essas conclusões estão baseadas em diversas análises empíricas encontradas na literatura, as quais apontam para a existência abundante de clusters 'não-dinâmicos', 'não-maduros', 'estáticos', 'em declínio', 'atrasados', 'quase-mortos', 'parados', 'mortos', entre outros (MALMBERG e MASKELL, 2002; MARTIN e SUNLEY, 2001; BEAUDY e BRESCHI, 2003; BATISTA e SWANN, 1998; BOSCHMA, 2004). 
Malmberg (2003) enumera algumas razões do descolamento entre a teoria de clusters e os estudos empíricos, a saber, a) as transações entre firmas dentro do cluster são geralmente limitadas e a colaboração é apenas formal; b) existe, de fato, algumas vezes, uma intensa rivalidade local; e c) as firmas do cluster armazenam bastante conhecimento útil acerca uma da outra, mesmo que estas freqüentemente não saibam onde este conhecimento se originou e se este é mesmo importante.

Em relação à cooperação entre firmas, a grande maioria dos estudos aponta para a existência abundante de conexões de cooperação verticais nas aglomerações, ou seja, ao longo da cadeia produtiva. De forma antagônica, os mesmos estudos ressaltam a irrelevância, ou mesmo a inexistência, de conexões de cooperação horizontais, exercidos por atores sem vínculos na cadeia produtiva, mostrando a escassez da cooperação horizontal (MALMBERG e POWER, 2003; SCHMITZ, 2000). Para Schmitz (2000), as pressões competitivas consistem na razão de haver cada vez menos cooperação horizontal.

Contrariando a bibliografia que parte do pressuposto Marshalliano, muitos estudos recentes sobre o tema criticam a forma como a abordagem de clusters vem sendo utilizada atualmente. Para Giuliani e Bell (2005), o conhecimento em um cluster não está livre no ar, mas é direcionado para as firmas que possuem maior capacidade de absorvê-lo. Schmitz (2000), Schmitz e Nadvi (1999), Malmberg (2003) e Giuliani et. al. (2005) criticam alguns estudos de clusters por apresentarem foco nas conexões internas. Para eles, o foco deve estar direcionado para as conexões externas (extracluster) para que se possa fazer uma análise de longo prazo e sustentável.

Outros estudos apontam mais algumas limitações da abordagem de clusters, tais como a carência de validação empírica satisfatória dos mecanismos teóricos apresentados, a ausência de um arcabouço teórico sólido e a ausência de uma teoria de clusters industriais, na qual o conhecimento seja o elemento central (MALMBERG, 1997; MASKELL e MALMBERG, 1999; MALMBERG e MASKELL, 2002). Schmitz e Nadvi (1999) ressaltam a necessidade de estudos voltados para comparações conclusivas entre clusters e direcionados para os aspectos relacionados ao conhecimento.

No entanto, Malmberg (2003) argumenta que, além das limitações da abordagem de clusters, existem várias razões pelas quais a proximidade geográfica consiste em fator importante no processo de transformação geral tanto das firmas quanto dos próprios aglomerados. Para ele o desafio, para entender como um cluster pode explorar e sustentar a competitividade internacional, é analisar como tais clusters podem desenvolver conexões para controlar as fontes de conhecimento especializado, onde quer que elas estejam no mundo. 


\section{A Abordagem de Sistemas de Inovação}

A abordagem de Sistemas de Inovação (SI) está relacionada, conforme o termo sugere, a 'sistemas' - que remete à idéia de complexidade das conexões e de diversidade de atores - e à 'inovação' - que remete à idéia de dinamismo e mudanças tecnológicas. O dinamismo está relacionado às mudanças tecnológicas, por meio das quais as firmas se adaptam e se modificam constantemente, buscando a sustentabilidade a longo prazo (EDQUIST, 1997; CARLSSON et. al., 2002).

Em relação à questão da delimitação geográfica, os estudos de SI podem apresentar uma flexibilidade de escolha, dependendo dos objetivos do estudo em questão. Assim, os estudos de SI podem:

- $\quad$ ser espacialmente delimitados, como (1) nacional (Sistemas Nacionais de Inovação SNI), (2) regional - supranacional ou sub-nacional (Sistemas Regionais de Inovação SRI) ou (3) local (Sistemas Locais de Inovação - SLI). A primeira possibilidade foca os limites nacionais de um sistema de inovação, a segunda os limites regionais (de um grupo de países ou de uma região dentro de um país) e a terceira os limites locais (i.e. uma área relativamente pequena, tal como uma cidade ou grupo de cidades); ou,

- apresentar uma estrutura espacialmente aberta, mas delimitada por: (1) uma dada tecnologia (Sistemas Tecnológicos de Inovação - STI), (2) por um determinado setor (Sistemas Setoriais de Inovação - SSI) ou (3) por uma dada corporação (Sistemas Corporativos de Inovação - SCI). A primeira foca uma tecnologia específica, a segunda foca um setor específico e a última foca uma corporação particular (no nível da firma).

Dentre as abordagens de SI discutidas anteriormente, utilizou-se as que dão ênfase aos sistemas setoriais e tecnológicos, por serem esses dois os mais aplicáveis ao caso estudado (aglomerado industrial da Bacia de Campos). A abordagem de sistemas setoriais de inovação (SSI), por analisar o sistema com foco no setor (desenvolvimento de campos de petróleo e gás em offshore) e a abordagem de sistemas tecnológicos de inovação (STI), por analisar o sistema com foco na tecnologia (tecnologias de poço e de equipamentos e sistemas submarinos de produção de petróleo e gás).

A abordagem dos Sistemas Setoriais de Inovação (SSI) complementa a abordagem dos sistemas de inovação (local, regional e nacional) e dos sistemas tecnológicos. Esta abordagem foca a inovação em um setor específico, ou seja, concentrado dentro dos limites setoriais. A abordagem de SSI usa uma visão multidimensional, integrada e dinâmica de setores a fim de analisar a inovação e tem origem no conceito de setor, tradicionalmente utilizado na economia industrial, porque este considera outros agentes que devem ser analisados além das firmas. Esta abordagem 
dispensa maior ênfase ao conhecimento, à aprendizagem e aos limites setoriais, foca as interações de não-mercado assim como as interações de mercado, e presta bastante atenção às instituições (MALERBA, 2004).

$\mathrm{Na}$ abordagem de SSI, a inovação, principal aspecto da análise, pode ser afetada por três fatores básicos (MALERBA, 2004: 18-28):

- Conhecimento e tecnologia: base particular de conhecimento, tecnologias e insumos do setor;

- Atores e redes: um setor consiste de agentes heterogêneos que são organizações ou indivíduos e as organizações podem ser firmas - usuários, produtores e fornecedores - ou não-firmas - universidades, organizações financeiras, agências governamentais, etc. As redes são elementos que caracterizam os relacionamentos entre os diferentes atores; e,

- Instituições: a cognição, as ações e as interações dos agentes são moldadas pelas instituições, que incluem normas, rotinas, hábitos comuns, leis, etc, diferentemente da noção intuitiva em relação ao termo.

A abordagem de Sistemas Tecnológicos de Inovação (STI) também deriva da abordagem de Sistema de Inovação (SI). Porém, diferentemente da abordagem de SSI, esta abordagem está centrada em uma tecnologia específica (enfatizando uma dada área tecnológica).

Os STI podem ser conceituados como rede ou redes de agentes interagindo em uma área tecnológica específica sob uma infra-estrutura institucional particular para gerar, difundir e utilizar tecnologia, onde os sistemas tecnológicos são definidos em termos dos fluxos de conhecimento e capacitações ao invés de fluxos de bens e serviços ordinários (CARLSSON, 1995). Os STI são compostos por um conhecimento dinâmico e por redes de capacitações tecnológicas (CARLSSON e STANKIEWICZ, 1991).

Carlsson (1995: 8) ressalta os componentes básicos da abordagem de STI como "primeiramente, a visão da tecnologia como conhecimento, e segundo, a conceituação de desenvolvimento tecnológico como um processo de resolução de problemas". O autor ainda aponta algumas características-chave dos STI, tais como: infra-estrutura tecnológica, redes de conhecimento e capacitações, massa crítica e competência econômica e o papel das políticas governamentais.

Essa abordagem, portanto, não considera aspectos relacionados com a territorialidade e proximidade geográfica como uma característica importante, da mesma forma que os SSI. Na realidade essa abordagem pode ser aplicada sob qualquer estratificação geográfica. Nesse sentido, apresenta uma gama flexível de aplicações e pode ser utilizada sob uma área geográfica local, regional ou até mesmo supranacional, ressaltando ainda que os STI são multidimensionais e, na maioria dos casos, os elementos que os constituem (redes de conhecimentos e capacitações, redes 
industriais e blocos de desenvolvimento e infra-estrutura institucional) são espacialmente correlacionados (CARLSSON, 1995).

No entanto, a abordagem de STI apresenta vantagens e desvantagens em relação aos SSI, o que terá maior ou menor relevância dependendo do foco de análise: a abordagem de STI foca em apenas uma tecnologia, a qual está relacionada a vários setores econômicos (cross-sectors), enquanto a abordagem de SSI foca em apenas um setor, o qual está relacionado a várias tecnologias (cross-technologies). É claro que a escolha de uma ou de outra abordagem específica está sempre baseada nos objetivos do estudo em questão e em suas perspectivas.

\section{Arcabouço Teórico e o Modelo Híbrido}

As estruturas de produção podem ser definidas como os projetos de produtos, materiais, máquinas, força de trabalho, e conexões de transação de mercado envolvidas na produção de bens e serviços em uma dada especificação. Por outro lado, todo o estoque de conhecimento dentro das firmas e os fluxos de conhecimentos entre as firmas e dentro das firmas, nos quais se baseiam as mudanças nos tipos de bens produzidos e nos métodos que são utilizados para produzi-los, é denominado de sistema de conhecimento (BELL e ALBU, 1999).

Para os autores, a descrição de uma firma, de um cluster ou de um setor, em termos de sua estrutura de produção, não diz muito sobre a evolução da firma ou do aglomerado que se analisa: sua história, trajetória atual ou capacidade para implementar mudanças tecnológicas no futuro. $\mathrm{O}$ sistema de conhecimento, por outro lado, "engloba os fluxos de conhecimento, estoques de conhecimento e sistemas organizacionais envolvidos em gerar e gerir mudanças em produtos, processos e organização da produção” (BELL e ALBU, 1999: 1723).

A importância do conhecimento e, conseqüentemente, dos sistemas de conhecimento, não parece ser contestada na literatura. É necessário ressaltar que tão importante quanto a existência de um sistema de conhecimento robusto é a capacidade de absorção desse conhecimento pelas firmas (MALERBA e ORSENIGO, 2000; GIULIANI, 2004).

Os sistemas de conhecimento e de produção podem ser caracterizados como abertos ou fechados. Bell e Albu (1999: 1721) ressaltam a importância de fontes internas e externas de conhecimento para um aglomerado: “em alguns casos, as fontes externas parecem exercer um papel maior ou até mesmo dominante. [...] Em outros casos, fontes de novas tecnologias extra-aglomerado perecem ter menos importância". Para eles, o sistema de conhecimento aberto para fontes externas de conhecimento tecnológico é possivelmente mais importante do que para fontes internas. Essa característica pode ser fundamental em setores como o de E\&P de petróleo e gás. Os autores discutem este ponto, mostrando que diversos estudos sugerem "que um sistema de conhecimento 
relativamente fechado pode estar associado à inabilidade de sustentar a competitividade a longo prazo" (BELL e ALBU, 1999: 1726).

Figura 1: Esquematização estrutural do Modelo Híbrido

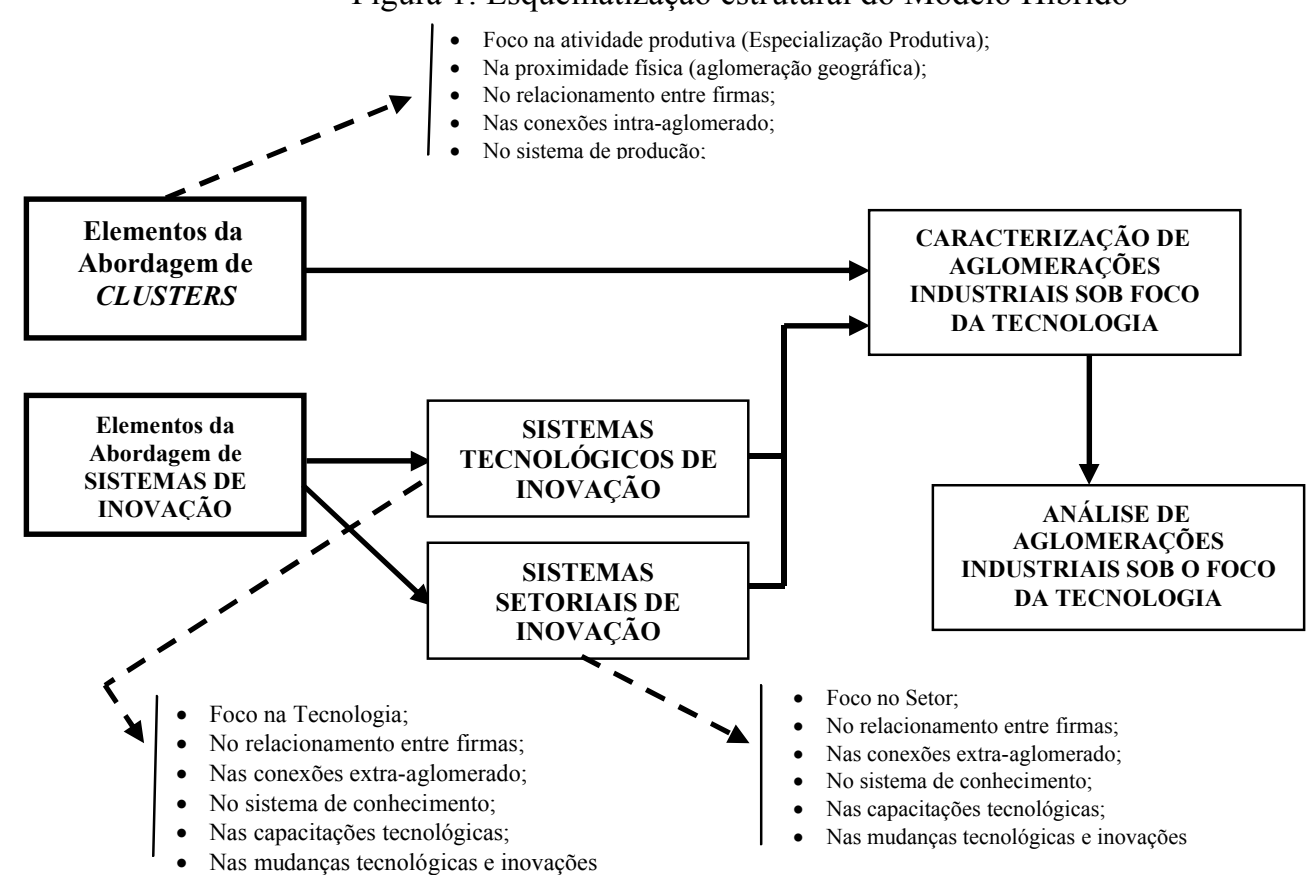

Fonte: Silvestre (2006)

Além disso, a importância das conexões extra-aglomerado fica evidente "desde que a ênfase em conhecimentos localizados pode resultar na 'morte entrópica' de um aglomerado que permanece fechado em uma trajetória tecnológica cada vez mais obsoleta" (GIULIANI e BELL, 2005). A existência de aglomerados estáticos, não desenvolvidos e atrasados tecnologicamente (que são mostrados extensivamente na literatura), reforça essa condição. Malmberg (2003: 159) corrobora esta idéia e reforça que as "interações em uma aglomeração são fascinantes e interessantes, mas entender conexões globais é pelo menos igualmente importante". Assim, a caracterização da estrutura do modelo híbrido está determinada na Figura 1, que estabelece a combinação dos elementos das duas abordagens discutidas, formando a base teórica para análise de aglomerações industriais.

\section{Considerações Finais}

A abordagem de cluster é geralmente utilizada em um contexto que apresenta algumas características específicas: estrutura do setor focada em atividades de manufatura, produção, aspectos industriais e conexões baseadas em fluxos de bens e serviços. Tais características são encontradas basicamente em aglomerados de produção/transformação de produtos manufaturados. Alguns exemplos são, entre outros, os setores de calçados, cerâmica de revestimento, tijolos, 
móveis, vinho, etc (SCHMITZ e NADVI, 1999; BELL e ALBU, 1999; GIULIANI, 2004). Por esta razão, pode-se afirmar que, na análise de clusters, o setor econômico em que este está inserido é relevante, ou seja, deve ser considerado obrigatoriamente na análise. Nesse caso, se o setor deve ser levado em conta, a diferença entre os setores pode representar uma importante característica a ser ressaltada em análises e, principalmente, comparações entre clusters industriais (PAVITT, 1984). Nessa situação, em aglomerações industriais de setores tecnologicamente dinâmicos, a abordagem de cluster pode apresentar algumas limitações.

Por esse motivo, da abordagem de sistemas de inovação, utiliza-se o elemento global (sem fronteiras geográficas definidas) e sistêmico. O termo 'sistêmico' introduz a diversidade de atores e a complexidade das relações e conexões como duas das principais características. 'Diversidade de atores' no sentido de analisar cuidadosamente, não somente as firmas, mas também o papel das diversas organizações que contribuem para o desenvolvimento das atividades do aglomerado (instituições de apoio), tais como: universidades, institutos de pesquisa, organizações reguladoras, organizações públicas, organizações de financiamento, etc. 'Complexidade das relações e conexões' no sentido de dar ênfase às relações e conexões intra-aglomerado (entre firmas ou entre firmas e organizações de apoio dentro do próprio aglomerado) e extra-aglomerado (entre firmas ou organizações situadas dentro do aglomerado com firmas ou organizações situadas fora do aglomerado - cross-bounderies).

Em que pese a incapacidade das abordagens existentes na literatura de apresentar, de forma isolada, uma estrutura analítica que satisfaça estudos em aglomerações industriais tecnologicamente dinâmicas, o Modelo Híbrido proposto pode ser ampliado e testado em outras aglomerações para lhe conferir validade podendo se transformar em uma ferramenta mais completa e um arcabouço teórico interessante para se entender as relações entre aspectos complexos como territorialidade, aprendizagem organizacional, capacitações tecnológicas, mudanças tecnológicas e inovações.

O referido Modelo foi aplicado com sucesso em estudo empírico realizado na aglomeração industrial de petróleo e gás da região produtora da Bacia de Campos gerando resultados promissores no entendimento dos aspectos supracitados (SILVESTRE, 2006).

No entanto, algumas limitações e dificuldades do estudo devem ser ressaltadas. Algumas delas são relativas ao arcabouço teórico utilizado e outras, relativas ao estudo empírico.

O tema tratado é recente e bastante dinâmico, com grande número de pesquisadores envolvidos. No entanto, essa dinâmica gera grande multiplicidade de nomenclaturas e conceitos, que, no fundo significam o mesmo tipo de estrutura ou estruturas bem próximas que emperram o desenvolvimento teórico e metodológico dos estudos na área.

No estudo empírico realizado, apesar das empresas estarem aglomeradas em um mesmo espaço geográfico e possuírem, grosso modo, a mesma especialização produtiva, apresentam outras 
características que diferem fortemente dos clusters tradicionais. O fato de haver jazidas de recursos naturais (petróleo e gás) abundantes na Bacia de Campos faz com que haja uma ausência de aspectos relacionados à cultura e tradição da região na atividade de E\&P de petróleo e gás.

O foco do estudo empírico está limitado à absorção de conhecimento tecnológico, para implementar as mudanças tecnológicas, por meio das conexões de conhecimento e das posturas tecnológicas das firmas. No entanto, sabe-se que existem outras formas de absorver conhecimento tecnológico, tais como: treinamento dos empregados, mobilidade da mão de obra, pesquisa e desenvolvimento dentro da firma (desenvolvimento endógeno), e outras, mas que não estão no foco central do estudo.

$\mathrm{O}$ acesso às firmas, como geralmente acontece, foi outra dificuldade encontrada ao longo do trabalho. A conciliação entre o tempo para elaboração do estudo e a disponibilidade limitada nas agendas dos executivos nem sempre é um problema simples de solucionar. Além disso, aspectos como concorrência, segredo industrial e pesquisa para inovação fazem com que os assuntos abordados neste trabalho não estejam na lista de preferência dos executivos para serem revelados a pessoas de fora da firma.

Finalmente, como sugestão para trabalhos futuros pode-se destacar:

- O aprimoramento e a consolidação do Modelo Híbrido, com a inserção de outras abordagens pertinentes (redes de firmas, ou outras);

- A aplicação da metodologia em outras aglomerações industriais, relacionadas a outras províncias petrolíferas;

- A aplicação da metodologia analítica em outros setores econômicos, como o de energia elétrica, automobilístico, aeronáutico, entre outros.

\begin{abstract}
This paper aims to present an innovative theoretical framework, elaborated to analyze industrial agglomerations which present high technological contents. A literature review of the clusters and innovations systems approaches was carried out, identifying the main problems of each one and their contributions to this dynamic and promissory research area. As main results of this work, a new theoretical model was carried out to analyze agglomerations, using elements from clusters and innovation systems approaches. From clusters approach, the relationships between actors are highlighted through their knowledge linkages, which together form the knowledge system. Concerning the innovation systems theory, elements from technological and sectoral approaches are used, trying to focus on the aspects related to the development of technological capabilities, technological changes and innovation. The model developed in this paper was applied on the oil and gas industrial agglomeration of the productive region of Campos Basin, generating promissory results.
\end{abstract}

Key-words: industrial agglomeration; clusters; innovation systems. 


\section{Referências}

AHARONSON, B. S.; BAUM, J. A. C.; FELDMAN, M. P. Industrial Clustering and the Returns to Inventive Activity: Canadian Biotechnology Firms, 1991-2000. DRUID Working Paper 04-03, 2004.

crossef

ALBU, M. Technological Learning and Innovation in Industrial Clusters in the South. Electronic Working Paper $\mathrm{n}^{\mathrm{o}}$ 7, SPRU, 1997.

AMIN, A. The potential for turning informal economies into marshallian industrial districts. in UNITED NATIONS: Technological dynamism in industrial districts. Geneva, 1994.

AUSDRETSCH, D. B.; FELDMAN, M. P. Innovative Clusters and the Industry Life Cycle. Review of Industrial Organization 11, pp. 253-273, 1996.

BASANT, R. Knowledge Flows and Industrial Clusters - An Analytical Review of Literature. Indian Institute of Management, Ahmedabad, 2002.

BATISTA, R.; SWANN, P. Do firms in Clusters innovate more? Research Policy 27, pp. 525-540, 1998. cross ${ }^{\text {ref }}$

BEAUDRY, C.; BRESCHI, S. Are firms in clusters really more innovative? Economics of Innovation and New Technologies, Vol. 12(4), pp. 325, 2003.

cross ${ }^{\text {ref }}$

BELL, M.; ALBU, M. Knowledge Systems and Technological Dynamism in Industrial Clusters in Developing Countries. World Development 27 (9): 1715. 1722-3.1726 1914, 1999.

BENDER, C.; HARMS, R.; RINDERMANN, G. Do Clusters Matter? Empirical Evidence from Germany's Neuer Markt. Paper presented at RENT XVI, Barelona, Nov. 2002 \& EIBA Conference, Athens, Dec. 2002.

BOSCHMA, R. Does geographical proximity favour innovation? Paper presented on $4^{\text {th }}$ Congress on Proximity Economics, Marseilles, 2004.

CARLSSON, B. (Ed.) Technological Systems and Economic Performance: The Case of Factory Automation Dordrecht, Kluwer, 1995.

CARLSSON, B.; JACOBSSON, S.; HOLMÉM, M.; RICKNE, A. Innovation Systems: Analytical and Methodological Issues. Research Policy 31, 233-245, 2002.

cross ${ }^{\text {ref }}$

CARLSSON, B.; STANKIEWICZ, R. On the Nature, Function, and Composition of Technological Systems. Journal of Evolutionary Economics, 1 (2), 93-118, 1991.

cross ${ }^{\text {ref }}$

CASSiOlATO, J. E.; LASTRES, H. M. M. Aglomerações, cadeias e sistemas produtivos e de inovações locais. Revista Brasileira de Competitividade, Ano 1, $\mathrm{n}^{\mathrm{0}} 1,2001$.

EDQUIST, C. Systems of innovation approaches: Their emergence and characteristics. in EDQUIST, C. (Ed.) Systems of innovation. Technologies, institutions and organizations. London: Pinter, 1997.

GIULIANI, E. When the micro shapes the meso: learning and innovation in wine clusters. DPhil Thesis, SPRU, University of Sussex, 2004.

GIULIANI, E.; BELL, M. The micro-determinants of meso-level learning and innovation: evidence from a Chilean wine cluster. Research Policy, vol. 34(1), pages 47-68, 2005.

cross ref 
GiUliani, E.; RABEllotTI, R.; VAN DIJK (Eds.) Clusters Facing Competition: The Importance of External Linkages. Ashgate Publishing, 2005.

HUMPHREY, J. Industrial reorganization in developing countries: from models to trajectories. World Development Vo1 23, No 1, 1995.

MALERBA, F. (Ed.) Sectoral Systems of Innovation - Concepts, Issues and Analyses of Six Major Sectors in Europe. Cambridge University Press, 2004.

MALERBA, F.; ORSENIGO, L. Knowledge, innovative activities and industry evolution. Industrial and Corporate Change, Vol. 9, n 2, 2000.

cross ${ }^{\text {ref }}$

MAlmberG, A. Industrial geography: location and learning. Progress in Human Geography, vol. 21, 1997.

MAlmberG, A. Beyond the Cluster - Local Milieus and Global Connections. in PECK, J.; YEUNG, W. (Eds.) Remaking the Global Economy - Economic-Geographical Perspectives. SAGE, 2003.

MALMBERG, A.; MASKELL, P. Towards an explanation of regional specialization and industry agglomeration. European Planning Studies, 5, 2541, 1997.

cross ${ }^{\text {ref }}$

MALMBERG, A.; MASKELL, P. The elusive concept of localization economies. Environment and Planning A, 34 : pp. 429-449, 2002.

cross ${ }^{\text {ef }}$

MALMBERG, A.; POWER, D. (How) do (Firms in) Clusters create Knowledge? Paper presented on DRUID Summer Conference, 2003.

MARCEAU, J. Clusters, Chains and Complexes: Three Approaches to Innovation with a Public Policy Perspective. in DOGSON, M.; ROTHWELL, R. (Eds.) The Handbook of Industrial Innovation. Edward Elgar, Cheltenhan, UK, 1994.

MARKUSEN, A. Sticky places in Slippery Space: A typology of Industrial Districts. Economic Geography, Vol. 72 , No. 3, 293-313, 1996.

crossef

MARSHALL, A. Principles of Economics. Eight Edition, Macmillan, London, UK, 1920.

MARTIN, R.; SUNLEY, P. Deconstructing clusters. Paper presented at the RSA Conference on Regionalising the Knowledge Economy, London, 2001.

MASKELL, P.; MALMBERG, A. Localised Learning and Industrial Competitiveness. Localised Journal of Economis 23, pp. $167-185,1999$.

PAVITT, K. Sectoral Patterns of Technical Change: Towards a Taxonomy and a Theory. Research Policy, Vol. 13, No. 6, pp. 343-373, 1984.

cross ${ }^{\text {ref }}$

PORTER, M. E. The Competitive Advantage of Nations. New York, Free Press, 1990.

SCHMITZ, H. Does Local Co-operation Matter? Evidence from Industrial Clusters in South Asis and Latin America. Oxford Development Studies, Vol. 28, No 3, 2000.

cross ${ }^{\text {ref }}$

SCHMITZ, H.; NADVI, K. Clustering and Industrialization: Introduction. World Development, Vol. 27, No. 9, pp. 1503-1514, 1999.

cross ref

SILVEStRe, B. S. Aglomeração Industrial de Petróleo e Gás da Região Produtora da Bacia de Campos: Conexões de Conhecimento e Posturas Tecnológicas das Firmas. Tese de Doutorado, DEI/PUC-Rio, 2006. 
STORPER, M. The regional world: Territorial development in a Global Economy. New York: Guilford, 1997.

\section{Dados dos autores:}

Bruno dos Santos Silvestre

Centrais Elétricas Brasileiras S. A. - ELETROBRÁS

Departamento de Novos Negócios

Supervisor de Negócios

Rua Álvaro Tâmega - 29, Centro, Campos dos Goytacazes - RJ, CEP: 28035-030; Brasil.

Telefones: (22) 9811-6645; (21) 2514-6064

e-mail: bruno.silvestre@eletrobras.comou bruss@ind.puc-rio.br

Paulo Roberto Tavares Dalcol

Pontifícia Universidade Católica do Rio de Janeiro - PUC-Rio

Departamento de Engenharia Industrial - DEI

Professor Associado

Rua Marquês de São Vicente, 225 - Gávea, Rio de Janeiro, RJ, CEP: 22453-900

Telefones: (21) 3527-1284, 3527-1285

e-mail:prtd@ind.puc-rio.br

Recebido para publicação em: 18/10/2006

Aceito para publicação em: 10/12/2006 